\title{
Stem Cell-Related Knowledge and Attitudes among Physicians in Jordan
}

\author{
Khalid Kheirallah ${ }^{1}$, Fayez Abdulrazeq ${ }^{2}$, Abdallah Alzoubi ${ }^{1}$, Jomana Alsulaiman ${ }^{3}$, Nasr \\ Alrabadi $^{1}$, Mahmoud Alfaqih ${ }^{1}$, Mazhar Al Zoubi ${ }^{4}$, and Monica Matsumoto ${ }^{5}$ \\ ${ }^{1}$ Jordan University of Science and Technology \\ ${ }^{2}$ JUST \\ ${ }^{3}$ Yarmouk University Faculty of Medicine \\ ${ }^{4}$ Yarmouk University \\ ${ }^{5}$ University of Chicago Pritzker School of Medicine
}

July 28, 2020

\begin{abstract}
Background: Rapid advancement of stem cell (SC) therapies provides both opportunities and risks for patients and physicians alike. Physicians have a role in counseling patients about unproven SC therapies, requiring a basic level of knowledge and access to information about SCs. Objective: This study sought to assess SC-related knowledge of and attitudes among physicians in Jordan to elucidate areas of deficiency that can be addressed. Methods: A cross-sectional survey, comprising questions on demographics and SC knowledge and attitudes, was designed as a scoring system to evaluate physicians' knowledge and attitudes. Participants were recruited from 10 major hospitals in Jordan over 3 months between February and April 2019. The internal consistency of the scoring scales was calculated using Cronbach's alpha reliability coefficient. Gender differences were evaluated with an independent t-test. Results: 382 physicians in Jordan completed the survey (59.9\% response rate). They demonstrated a low/moderate level of overall SC knowledge (51.3\%), but most lacked confidence in their ability to answer patients' questions about SC therapies (64.7\%). However, the total attitude score was moderate/high positive (66.8\%) and most were interested in learning more about SCs (80.8\%). Male physicians reported significantly more knowledge than females $(\mathrm{p}<0.0001)$. Conclusions: This study reveals Jordanian physicians' hesitancy to counsel patients about SC therapies, largely due to gaps in knowledge. However, overall attitudes toward SC research and therapies are positive. The results of this study demonstrate a need to cover SC-related information in medical curricula in Jordan, as well as to support initiatives to regulate $\mathrm{SC}$ tourism in Jordan.
\end{abstract}

\section{Hosted file}

Manuscript V1.docx available at https://authorea.com/users/337115/articles/472742-stem-cellrelated-knowledge-and-attitudes-among-physicians-in-jordan 